\title{
Recent advances of Wee1 inhibitors and Statins in gynecologic cancers with p53 mutations
}

Xiangbing Meng ${ }^{1,2^{*}}$, Jason Z Gao ${ }^{1}$, Sean Michael T Gomendoza ${ }^{1}$, John $\mathrm{Li}^{1,3}$, Shujie Yang ${ }^{1,2^{*}}$

${ }^{1}$ Department of Pathology, ${ }^{2}$ Holden Comprehensive Cancer Center, Carver College of Medicine, University of Iowa, ${ }^{3}$ University of Southern California.

*Corresponding authors.

\section{Abstracts}

p53 is among the most frequently mutated tumor suppressor genes given its prevalence in $>50 \%$ of all human cancers, including high grade serous endometrial cancers and ovarian cancers. In addition to loss of tumor suppression function, many mutated p53 (Mutp53) proteins acquire gain-of-function (GOF) activities as oncogenes to promote cancer progression, which manifest through aberrant expression of p53. As we have come to see, statins induce CHIP-mediated degradation of mutp53 by blocking the interaction between mutp53 and DNAJA1. Therefore, targeting critical downstream pathways of mutp53 provides an alternative strategy for treating cancers expressing mutp53. In this review, we summarize recent advances with Wee1 inhibitors and mevalonate pathway inhibitors, particularly statins, regarding their use in gynecological cancers with p53 mutations.

Keywords: p53, Wee1, AZD1775, adavosertib, statins.

\section{Introduction}

Over $50 \%$ of all tumors harbor mutations in the p53 gene, demonstrating its criticality in tumor development. p53 is activated by a wide variety of stress signals and selectively transcribes a set of downstream target genes by acting as a transcription factor to initiate various cellular responses to exert its function in tumor suppression. Although the roles of p53 in regulation of cell cycle arrest, senescence and apoptosis have been widely discussed, the role of p53 in the regulation of ferroptosis and anti-oxidant defense is also critical for its tumor suppressive function. Unlike p53 null mice, which rapidly and spontaneously develop thymic lymphomas, no tumor development is observed in p53(3KR/3KR) animals bearing lysine to arginine mutations at p53 acetylation sites of K117R,K161R and K162R. p53(3KR), an acetylation-defective mutant that lacks activities in cell-cycle arrest, senescence and apoptosis, fully retains the activity to repress SLC7A11 expression and induce ferroptosis in reactive oxygen species (ROS)induced stress (1-3). Analysis of mutant mice with p53(3KR) shows that these non-canonical p53 activities 
contribute to embryonic development and the lethality associated with loss of Mdm2. It is well established that ferroptosis is primarily controlled by inhibiting GPX4. p53 activation modulates ferroptosis without apparent effects on glutathione peroxidase 4 (GPX4) function. Instead, ALOX12 is critical for p53-mediated ferroptosis. ALOX12 inactivation diminishes p53-mediated ferroptosis induced by ROS and abrogates p53dependent inhibition of tumor growth in xenograft models. The Gu lab reported an ALOX12-mediated, GPX4/ACSL4-independent ferroptosis pathway for p53-dependent tumor suppression (1-3). p53 inhibits cystine uptake by repressing expression of SLC7A11, a key component of the cystine/glutamate antiporter and sensitizes cells to ferroptosis, which is highly expressed in human tumors. ALOX12 missense mutations abrogate its ability to oxygenate polyunsaturated fatty acids and to induce p53-mediated ferroptosis in human cancers. The ALOX12 gene is located at human chromosome 17p13.1, a hotspot of monoallelic deletion sites in cancers. Many tumor hotspot mutants p53 not only lose tumor-suppressive functions, but also gain new activities in promoting tumorigenesis, called mutant p53 gain-of-function (GOF). Mutp53 not only promotes tumor development, but also renders these cancer cells with mutp53 vulnerable to some downstream pathways which they are dependent on for survival as descripted in many reviews (4-6). The progress of Wee1 inhibitors and statins in gynecological cancer with p53 mutations will be discussed.

\section{Wee1 inhibitors}

Tumor suppressor $\mathrm{p} 53$ plays a critical role in cell cycle arrest at G1/S and G2/M in response to DNA damage to prevent DNA replication collapse and mitotic catastrophe. Tumor cells with absent or mutated p53 are vulnerable to agents targeting the G2/M checkpoint due to the loss of the G1/S checkpoint. Overriding the $\mathrm{G} 2$ checkpoint will preferentially sensitize p53-defected tumor cells to DNA-damaging agents and spare normal cells with wild type p53, which provides a therapeutic window for cancer cells with p53 defects. Wee1 and Cdc25 regulate cdc2 activity by modifying phosphorylation at Tyr15 at the G2/M checkpoint and preventing entry into mitosis. The overexpression of WEE1 has been observed in several cancer types, including ovarian cancer. The WEE1 inhibitor AZD1775 (also called adavosertib and MK1775) can override the $\mathrm{G} 2 / \mathrm{M}$ checkpoint by activating cdc2 via preventing cdc2 phosphorylation at tyrosine 15(Figure 1A). AZD1775 was observed to promote a synergistic cytotoxicity with the chemotherapeutic agent gemcitabine or the PARP1 inhibitor olaparib in p53-mutant ovarian and endometrial cancer cells (7). Diminishing drug resistance to carboplatin via treatment with AZD1775 was observed in p53-mutant tumors resistant to first-line platinum-based therapy in a phase II clinical trial (8). GOF mutp53 was found to increase the expression of proteasome genes to protect against proteotoxic stress, conferring 
resistance to proteasome inhibitors (9). Poly (ADP-Ribose) Polymerase (PARP) plays a critical role in maintenance genomic stability; as such, inhibition of PARP coincident with Wee1 inhibitors is theorized to synergistically reduce cells passing freely through the G2 DNA damage checkpoints. However, simultaneous induction of both treatments, although effective in inhibiting tumor growth, is offset by a strong toxicity and low tolerance characterized by weight loss and anemia by the end of a month-long treatment period. Fang et al. recently reported that the synergistic nature of PARP and Wee1 inhibitors was maintained while minimizing toxicity when sequential rather than concurrent therapies were employed in ovarian cancer xenograft and patient-derived xenograft models(10). These were significantly enhanced from the original monotherapeutic strategies and largely on par with concurrent outcomes to create reduced fork speed, increased DNA damage, and consequent slowed cell growth relative to normal cells based on endogenous replication stress levels and increased cell cycle arrest at the G1/S and G2/M checkpoints. To increase specificity to Wee1 and reduce toxicity to normal cells, the Wee1 kinase degrader, ZNL-02-096, was developed by binding AZD1775 to the cereblon (CRBN)-binding ligand pomalidomide by using PROTAC technique. Degradation upon conjugation of AZD1775 to pomalidomide functioned in a CRBN and proteasome-dependent manner, creating the novel and notably low-dose mechanism by which WEE1 overexpression in cancer cells may be repressed. ZNL-02-096 has recreated mono-therapeutic AZDG2/M arrest rates at a significant 10 -fold dosage decrease, thereby minimizing dosage-dependent toxicity while maintaining WEE1 inhibition efficacy and specificity. In particular, the secondary target PLK1 was avoided, indicating the reduction of blood-related side effects of this Wee1 degrader when used to treat patients in clinical trials. 


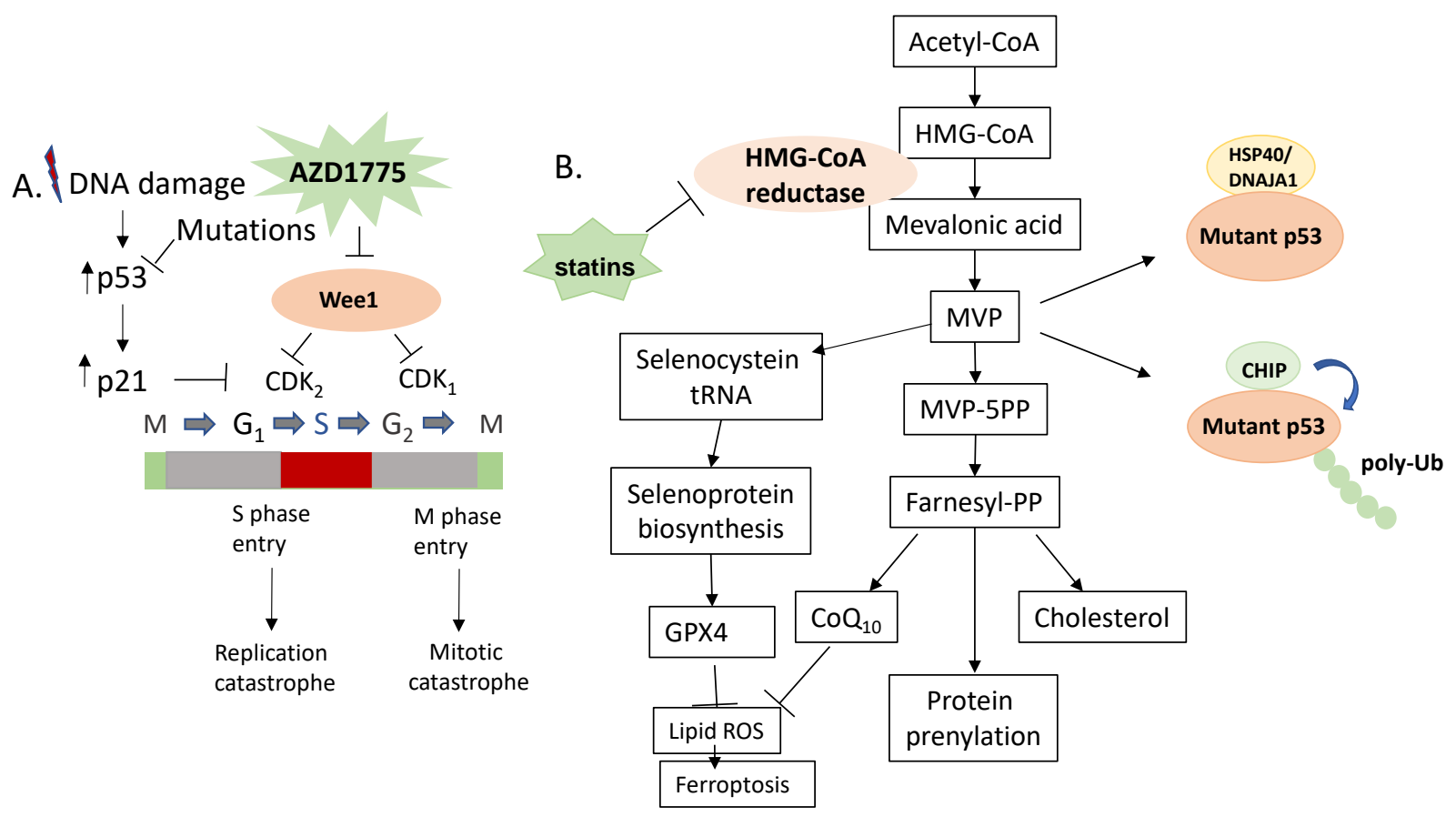

Figure 1. Therapeutic strategies targeting Wee1 and the mevalonate pathway in cancer with p53 mutations. The therapeutic strategies targeting mutp53 in cancer include targeting mutp53 directly or indirectly. The indirect strategies include inhibition of the critical downstream pathways of mutp53 and the direct strategies include inducing mutp53 degradation. (A) Wee1 inhibitor induces replication catastrophe and mitotic catastrophe in cancer cells with p53 mutations. (B). Statins inhibit mevalonate pathway and promote degradation of mutp53. Mevalonate-5-phosphate (MVP) promotes mutp53 stabilization by increasing the interaction between mutp53 and KSP40/DNAJA1 and inhibiting CHIP-mediated ubiquitination and proteasomal degradation of mutp53. Inhibition of the mevalonate pathway with statins represents a novel therapeutic strategy of targeting cancers with mutp53 by inducing mutp53 degradation, inhibiting prenylation of oncogenes and cholesterol synthesis, as well as inducing ferroptosis by inhibiting the biosynthesis of GPX4 and CoQ10.

The single-agent activity of AZD1775/adavosertib was tested in paired tumor biopsies of patients carrying BRCA mutations as proof-of-mechanism to demonstrate the target modulation and DNA damage response at clinical trial NCT01748825 (11). AZD1775 was demonstrated to be safe and tolerable as a single agent and in combination with chemotherapy at the clinical trial NCT00648648 (8). The $21 \%$ response rate in TP53-mutated patients $(n=19)$ was higher than the 12\% in TP53 wild-type patients $(n=$ 33), indicating that AZD1775 is effective at both TP53 wild-type and TP53-mutated patients. Adavosertib monotherapy demonstrated encouraging and durable evidence of activity in women with recurrent uterine serous carcinoma (USC). Liu et al. reported a phase II single-arm clinical trial (NCT03668340) of adavosertib in 34 patients with recurrent or persistent Uterine Serous Carcinoma (12). Median PFS was 
6.1 months, and median duration of response was 9.0 months. Adverse events (AEs) included diarrhea (76.5\%), fatigue (64.7\%), nausea (61.8\%), anemia (67.6\%), low platelet count (17.6\%), or low neutrophil count (32.4\%). (13) Progression-free survival (PFS) was longer with adavosertib plus gemcitabine (median 4.6 months vs 3.0 months with placebo plus gemcitabine in platinum-resistant or platinum-refractory recurrent ovarian cancer patients at the phase 2 trial NCT02151292 (14). Adding adavosertib to chemotherapy improved PFS [median, 7.9 vs. 7.3 months in patients with TP53-mutated, platinumsensitive ovarian cancer treated with adavosertib $(A+C)$ plus carboplatin and paclitaxel compared to placebo plus carboplatin and paclitaxel group $(\mathrm{P}+\mathrm{C})$ in the phase II clinical trial NCT01357161. An increase in adverse events of adavosertib was observed in patients treated with $A+C$ versus $P+C$ : greatest for diarrhea (75\%; 37\%), vomiting (63\%; 27\%), anemia (53\%; 32\%), and all grade $\geq 3$ adverse events (78\%; 65\%). PMID: 32611648 Additional adavosertib doses within the chemotherapy treatment cycle or the potential for maintenance therapy for adavosertib should be considered to increase clinical benefit in future studies.

Clinical activity of ZN-c3, a potent oral WEE1 inhibitor from Zentalis Pharmaceuticals, in a Phase 1 doseescalation trial was recently reported in 55 patients with advanced or metastatic solid tumors at the AACR Annual Meeting 2021. Better safety results were observed compared to adavosertib and partial responses in five patients were observed. The drug's safety profile could make it particularly well suited for use in combinations. Side effects were mostly mild to moderate, with nausea affecting about half of the 55 patients evaluable for safety, and diarrhea, fatigue and vomiting afflicting less than one-third of them. Of note, blood-related side effects struck less than $10 \%$ of patients: $1.8 \%$ of patients suffered a low white blood cell count, $7.2 \%$ of patients had a low platelet count and $7.2 \%$ of patients developed anemia. There are three clinical trials for $\mathrm{ZN}-\mathrm{c} 3$ relevant to gynecological cancer, including a study of $\mathrm{ZN}-\mathrm{c} 3$ in patients with solid tumors (NCT04158336), a study of ZN-c3 in patients with platinum-resistant ovarian cancer (NCT04516447) and a study of ZN-c3 in women with recurrent or persistent uterine serous carcinoma( NCT04814108). For the clinical trial in NCT04516447 in a study of ZN-c3 in patients with platinum-resistant ovarian cancer, levels of circulating deoxyribonucleic acid TP53 mutations tested by TAm-Seq will be correlated with response and changes in pCDC2 and pH2AX in skin and tumor tissue will be evaluated as pharmacodynamic markers of therapy. At clinical trial NCT04158336, ZN-c3 will be tested to treat patients with solid tumors with advanced or metastatic disease as a single agent and in combination with PARP inhibitor Talazoparib or PD1 inhibitor Pembrolizumab. 
Wee1 inhibitor treatments were also found to promote cancer immunotherapy. Co-targeting of WEE1 and DNA damage response kinase ATM was shown to downregulate PD-L1 expression in pancreatic cancer. (15) WEE1 inhibition was found to sensitize cancer cells to immunotherapy to PD-1 checkpoint blockade in oral cavity carcinoma, melanoma and colon adenocarcinoma with variable Tp53 mutations, which provide a pre-clinical rationale for the combination of agents that target cell cycle checkpoints and activate anti-tumor immunity to support the clinical trials of Wee1 inhibitor in combination with immunotherapy. (16). The effect of the combination of Wee1 inhibitor ZN-c3 with PD1 inhibitor Pembrolizumab will be studied in patients with solid tumors with advanced or metastatic disease at the clinical trial NCT04158336.

In addition to p53 mutations, overexpression of SKP2 and CUL1 at cancer patients may predict benefit to Wee1 inhibitors. Overexpression of G1/S regulatory genes, including SKP2, CUL1, and CDK2, was identified as resistance mechanisms to WEE1 inhibitor in a genome-wide unbiased genetic screen (17). Stable depletion of SKP2, CUL1, or CDK2 rescued sensitivity to Wee1 inhibition in breast and ovarian cancer cell lines, indicating that cancer patients with overexpression of these $\mathrm{G} 1 / \mathrm{S}$ regulatory genes would respond well to Wee1 inhibitor.

2. Mutant p53 and mevalonate pathway

Missense mutations in the TP53 gene can lead to the accumulation of dysfunctional TP53 (mutp53) proteins, which have gain-of-function activities that include activation of SREPB transcription factors (specifically SREBP2), which upregulates mevalonate pathway enzymes. This, in turn, leads to tumor growth and progression. However, these dysfunctional TP53 proteins must first be stabilized to have these effects. As it turns out, the mevalonate pathway itself fulfills this role (18). The mevalonate pathway is critical in facilitating tumor proliferation as it produces necessary sterols and isoprenoids from acetyl CoA (19). These compounds, especially isoprenoids, are required for processes such as protein prenylation and lipidation that enable Ras and Rho GTPases to anchor to the cell membrane, both of which function in cell proliferation (18). Though there are many intermediates and reactions found within the mevalonate pathway, the action of HMG CoA reductase has been particularly of interest as it is believed to be regulated by various other studied factors. Statins are a class of cholesterollowering drugs to inhibit $\mathrm{HMG}$ CoA reductase used to prevent cardiovascular disease including 
atherosclerosis and coronary heart disease in people at high risk.

As previously mentioned, mutp53 stimulates the mevalonate pathway by binding to SREBP2, which is a transcription factor that p53 itself activates. SREBP2 in turn affects cellular location and activates YAP and TAZ, both of which have previously been seen to function as potent oncogenes while also mediating the Hippo pathway, which prioritizes cell proliferation and survival $(20,21)$. In addition, mutp53 also interacts with nuclear factor Y (NF-Y), which functions to increase expression of the Rho family of small GTPases, which also function in cell proliferation. Interestingly, recent studies have provided evidence to believe that mutp53, through inducing SREBP2 and associated genes/pathways as well as NF-Y, can potentially transcriptionally activate HMG CoA reductase, thereby upregulating the mevalonate pathway as a whole and promoting survival pathways (20).

Additionally, these statins have minimal effects on WT p53 and DNA contact mutants, which offers the advantage of reducing side effects to normal cells with wild type p53 (Figure 1B). Statins accomplish this function by reducing mevalonate-5-phosphate (MVP), a metabolic intermediate in the mevalonate pathway (22). This triggers CHIP ubiquitin ligase-mediated degradation by disrupting the binding affinity between mutp53 and DNAJA1, a Hsp40 family member. Since mutp51 cannot bind or interact with DNAJA1, it will instead bind to CHIP and undergo degradation. The actual mechanism through which this interruption of mutp53-DNAJA1 interaction after reduction of MVP occurs is not entirely known but is hypothesized to be due to subsequent changes in protein folding machinery or post-translational modifications that affect DNAJA1 and/or mutp53. To further support the significance of DNAJA1 to mutp53 function, it was later found that knockout of DNAJA1 can also induce CHIP-mediated mutp53 degradation while overexpression antagonizes statin-induced mutp53 degradation (22). This latter effect is of particular interest because statin treatment is a method that is particularly effective against conformational mutp53 as statins inhibit mutp53 stabilization and protein prenylation, both of which are critical to mutp53's ability to carry out its effects. It was later determined that knockout of mevalonate kinase (MVK) has the same effect as reducing mevalonate 5-phosphate, suggesting that the disruption of mutp53 functions may be brought about through targeting and manipulating different parts of the mevalonate pathway (18). In addition to its effects on mevalonate 5-phosphate, statins inhibit HMG-CoA (HMGCR) reductase activity, which mediates the synthesis of 
cholesterol and the inhibition of the biosynthesis of selenoproteins (such as GPX4) and CoQ10, and thus enhance ferroptosis $(22,23)$. Several groups reported that Hsp90, Hsp40, CHIP and MDM2 plays critical roles to stabilize mutant p53 by the HSP chaperone system, suggesting possible synergism between Hsp90 inhibitors and statins or Hsp90 and Hsp40 inhibitors in combination $(22,24-26)$.

To support this previous information, it is worth mentioning that the gene signature of the activation of the mevalonate pathway was identified in an orthotopic model of epithelial ovarian cancer with the p53 mutation in 2016 (20). This model utilized aggressive abdominal ascitesderived 28-2 cells, which were found to have many upregulated genes of the mevalonate pathway. As expected, this upregulation had an association with the acquisition of the p53 mutation. Consistent with what was previously mentioned, treatment of these cells with a statin, simvastatin in this case, induced apoptosis in these cells via inhibition of HMG CoA reductase, the ratelimiting step of the mevalonate pathway that normally produces an intermediate that is converted into mevalonate 5-phosphate via mevalonate kinase (20). As an added note, it was found that 282 cells were more sensitive than other parental cell lines (such as ID8) to statin treatment and that simvastatin-induced cell death could only be rescued by mevalonate and not by cholesterol, demonstrating the significance of interrupting the mevalonate pathway in dealing with mutp53 (20).

As of now, there are over 100 recorded clinical trials that implement statin treatment in cancerrelated scenarios, including gynecological cancers. For instance, atorvastatin was used in the phase II cancer prevention clinical trial NCT04767984 in treating patients with ulcerative colitis who have dominant-negative missense p53 mutations and are at risk of developing large intestinal cancer. Atorvastatin was also used in pilot trial NCT03560882, which will hopefully determine if atorvastatin given at a dose of 80 milligrams per day (mg/day) for 1 to 4 weeks can decrease the level of conformational mutant p53, Ki-67 and increase caspase-3 in patients with solid tumor and relapsed acute myeloid leukemia (AML). In gynecological cancers, there are several clinical trials involving statin treatment in ovarian cancer patients and endometrial cancer patients. The clinical trial NCT04491643 will explore the treatment efficacy of megestrol acetate 160mg plus rosuvastatin 10mg by mouth daily for 6 months in patients with early endometrial carcinoma (EEC) seeking for conservative treatment by hysteroscopy at every 3 months. The preoperative window, 
phase 0 study of trial NCT02767362 will evaluate anti-proliferative effects of atorvastatin for 2 to 4 weeks treatment by measuring Ki67 immunohistochemical staining in obese women who are to undergo surgical staging for endometrial cancer. The clinical trial NCT04457089, yet another example, is a single arm pilot trial to evaluate of the effect of simvastatin at $40 \mathrm{mg}$ daily for approximately 6 months on cancer progression and change in serum level of CA125 among patients with platinum-sensitive ovarian cancer, treated with carboplatin and liposomal doxorubicin at Cedars-Sinai Medical Center. These platinum-sensitive ovarian cancer patients are at high risk of developing recurrent disease and have the potential to get the maximum benefit from simvastatin. Another clinical trial, NCT00585052, will evaluate if the treatment combination of paclitaxel and lovastatin is more effective for patients with refractory or relapsed ovarian cancer. Other than this, there are 3 recorded trials investigating the role of TP53 at statins associated treatment to cancer. The clinical trial NCT02767362 will study the effect of atorvastatin for a minimum of 2 weeks with a dosage of $80 \mathrm{mg}$ once, daily on endometrial cancer. In clinical trial NCT03560882, atorvastatin will be used for a period of 1-4 weeks with a dosage of $80 \mathrm{mg}$ per day to see efficiency and potency of atorvastatin and the effect on the reduction of mutant p53. In trial NCT04767984, atorvastatin will be investigated on its effect in reducing mutant p53 levels in patients. These trials demonstrate remarkable effort and drive in an attempt to see if statins can be re-purposed against the effects of mutp53 by blocking the mevalonate pathway in cancer patients with TP53 mutations.

\section{Concluding remarks}

As summarized above, Wee1 inhibitor and statins have shown to be effective to certain extent and promising in both preclinical studies and clinical trials to gynecologic cancer with p53 mutations; however, there are still unresolved obstacles. More studies on the application of Wee1 inhibitors and statins as monotherapy or combined with other reagents in p53 mutated cancers will improve therapeutics efficacy mutp53 in gynecologic cancers.

\section{Funding}

This manuscript was supported by NIH grants R01CA184101 (XM) and R37CA238274 (SY). XM was also supported by an Oberley award from HCCC. HCCC at The University of Iowa was supported by National Cancer Institute Award P30CA086862.

\section{Conflict of interest: none declared.}




\section{References}

1. Chu B, Kon N, Chen D, Li T, Liu T, Jiang L, et al. ALOX12 is required for p53-mediated tumour suppression through a distinct ferroptosis pathway. Nature cell biology. 2019;21(5):579-91.

2. Jiang L, Kon N, Li T, Wang SJ, Su T, Hibshoosh H, et al. Ferroptosis as a p53-mediated activity during tumour suppression. Nature. 2015;520(7545):57-62.

3. Li T, Kon N, Jiang L, Tan M, Ludwig T, Zhao Y, et al. Tumor suppression in the absence of p53mediated cell-cycle arrest, apoptosis, and senescence. Cell. 2012;149(6):1269-83.

4. Zhang C, Liu J, Xu D, Zhang T, Hu W, Feng Z. Gain-of-function mutant p53 in cancer progression and therapy. J Mol Cell Biol. 2020;12(9):674-87.

5. Zhu G, Pan C, Bei JX, Li B, Liang C, Xu Y, et al. Mutant p53 in Cancer Progression and Targeted Therapies. Front Oncol. 2020;10:595187.

6. Schulz-Heddergott R, Moll UM. Gain-of-Function (GOF) Mutant p53 as Actionable Therapeutic Target. Cancers (Basel). 2018;10(6).

7. Meng X, Bi J, Li Y, Yang S, Zhang Y, Li M, et al. AZD1775 Increases Sensitivity to Olaparib and Gemcitabine in Cancer Cells with p53 Mutations. Cancers (Basel). 2018;10(5).

8. Leijen S, van Geel RM, Pavlick AC, Tibes R, Rosen L, Razak AR, et al. Phase I Study Evaluating WEE1 Inhibitor AZD1775 As Monotherapy and in Combination With Gemcitabine, Cisplatin, or Carboplatin in Patients With Advanced Solid Tumors. J Clin Oncol. 2016;34(36):4371-80.

9. Walerych D, Lisek K, Sommaggio R, Piazza S, Ciani Y, Dalla E, et al. Proteasome machinery is instrumental in a common gain-of-function program of the p53 missense mutants in cancer. Nature cell biology. 2016;18(8):897-909.

10. Fang Y, McGrail DJ, Sun C, Labrie M, Chen X, Zhang D, et al. Sequential Therapy with PARP and WEE1 Inhibitors Minimizes Toxicity while Maintaining Efficacy. Cancer Cell. 2019;35(6):851-67 e7.

11. Mueller S, Haas-Kogan DA. WEE1 Kinase As a Target for Cancer Therapy. J Clin Oncol. 2015;33(30):3485-7.

12. Liu JF, Xiong N, Campos SM, Wright AA, Krasner C, Schumer S, et al. Phase II Study of the WEE1 Inhibitor Adavosertib in Recurrent Uterine Serous Carcinoma. J Clin Oncol. 2021;39(14):1531-9.

13. Uterine Serous Carcinoma Responds to the WEE1 Inhibitor Adavosertib. Cancer discovery. 2021;11(5):OF27.

14. Lheureux S, Cristea MC, Bruce JP, Garg S, Cabanero M, Mantia-Smaldone G, et al. Adavosertib plus gemcitabine for platinum-resistant or platinum-refractory recurrent ovarian cancer: a double-blind, randomised, placebo-controlled, phase 2 trial. Lancet. 2021;397(10271):281-92.

15. Jin MH, Nam AR, Park JE, Bang JH, Bang YJ, Oh DY. Therapeutic Co-targeting of WEE1 and ATM Downregulates PD-L1 Expression in Pancreatic Cancer. Cancer Res Treat. 2020;52(1):149-66.

16. Sun L, Moore E, Berman R, Clavijo PE, Saleh A, Chen Z, et al. WEE1 kinase inhibition reverses $\mathrm{G} 2 / \mathrm{M}$ cell cycle checkpoint activation to sensitize cancer cells to immunotherapy. Oncoimmunology. 2018;7(10):e1488359.

17. Heijink AM, Blomen VA, Bisteau X, Degener F, Matsushita FY, Kaldis P, et al. A haploid genetic screen identifies the $\mathrm{G} 1 / \mathrm{S}$ regulatory machinery as a determinant of Wee1 inhibitor sensitivity. Proceedings of the National Academy of Sciences of the United States of America. 2015;112(49):151605.

18. Parrales A, Thoenen E, Iwakuma T. The interplay between mutant p53 and the mevalonate pathway. Cell death and differentiation. 2018;25(3):460-70.

19. Mullen PJ, Yu R, Longo J, Archer MC, Penn LZ. The interplay between cell signalling and the mevalonate pathway in cancer. Nat Rev Cancer. 2016;16(11):718-31. 
20. Greenaway JB, Virtanen C, Osz K, Revay T, Hardy D, Shepherd T, et al. Ovarian tumour growth is characterized by mevalonate pathway gene signature in an orthotopic, syngeneic model of epithelial ovarian cancer. Oncotarget. 2016;7(30):47343-65.

21. Pan D. The hippo signaling pathway in development and cancer. Dev Cell. 2010;19(4):491-505.

22. Parrales A, Ranjan A, lyer SV, Padhye S, Weir SJ, Roy A, et al. DNAJA1 controls the fate of misfolded mutant p53 through the mevalonate pathway. Nature cell biology. 2016;18(11):1233-43.

23. Chen X, Kang R, Kroemer G, Tang D. Broadening horizons: the role of ferroptosis in cancer. Nat Rev Clin Oncol. 2021;18(5):280-96.

24. Ingallina E, Sorrentino G, Bertolio R, Lisek K, Zannini A, Azzolin L, et al. Mechanical cues control mutant p53 stability through a mevalonate-RhoA axis. Nature cell biology. 2018;20(1):28-35.

25. Li D, Marchenko ND, Schulz R, Fischer V, Velasco-Hernandez T, Talos F, et al. Functional inactivation of endogenous MDM2 and CHIP by HSP90 causes aberrant stabilization of mutant p53 in human cancer cells. Mol Cancer Res. 2011;9(5):577-88.

26. Sener A, Malaisse-Lagae F, Malaisse WJ. Stimulation of pancreatic islet metabolism and insulin release by a nonmetabolizable amino acid. Proceedings of the National Academy of Sciences of the United States of America. 1981;78(9):5460-4. 